\title{
Prevalencia de síntomas del reflujo gastroesofágico y factores asociados: una encuesta poblacional en las principales ciudades de Colombia
}

\section{Prevalence of Gastro-Esophageal Reflux Symptoms and Associated Factors: A Population Survey in the Principal Cities Of Colombia}

David B. Páramo-Hernández, MD, ${ }^{1}$ Rosario Albis, MD, MSc, ${ }^{1}$ María T. Galiano, MD, ${ }^{1}$ Belén de Molano, MD, ${ }^{1}$ Reynaldo Rincón, MD, ${ }^{1}$

Luis F. Pineda-Ovalle, MD, ${ }^{1}$ Alberto Rodríguez, MD, ${ }^{1}$ William Otero Regino, MD, ${ }^{1}$ Albis Hani, MD, ${ }^{1}$ Luis Carlos Sabbagh, MD, ${ }^{1}$

Carolina Sandoval-Salinas, MSc, ${ }^{1}$ Ricardo Sánchez-Pedraza, MD, MSc, ${ }^{1}$

Comité de Investigaciones, Asociación Colombiana de Gastroenterología. Bogotá, Colombia.

Fecha recibido: $29-02-16$ Fecha aceptado: 01-11-16

\section{Resumen}

Problema: el reflujo gastroesofágico es un proceso fisiológico que en algunas personas puede tornarse patológico y que produce molestias y lesiones esofágicas y extraesofágicas que afectan la calidad de vida de los individuos que lo presentan. Actualmente no se cuenta con estadísticas sobre la frecuencia de esta condición en Colombia. Objetivo: cuantificar la prevalencia de síntomas de enfermedad por reflujo gastroesofágico (ERGE) en los adultos de cuatro ciudades principales de Colombia utilizando el cuestionario GERDQ, y explorar la asociación de variables sociodemográficas y antropométricas con esta enfermedad.

Metodología: estudio de corte transversal de base poblacional. Se incluyeron 6842 personas, mayores de 18 años y menores de 80 años de edad, residentes en Bogotá, Cali, Medellín y Barranquilla. La muestra fue seleccionada por muestreo aleatorio estratificado. Se aplicó una encuesta telefónica que incluía el cuestionario GERDQ para la identificación de síntomas de reflujo en la última semana. Se calculó la prevalencia de reflujo por estratos teniendo en cuenta el punto de corte de la encuesta (8 o más puntos) y se realizaron comparaciones entre ellos utilizando la prueba de $x^{2}$. Se exploró la asociación de variables sociodemográficas y antropométricas mediante análisis bivariados y modelos de regresión logística.

Resultados: se obtuvieron 6842 encuestas. La prevalencia estimada de síntomas de reflujo en general fue del 11,98\% (IC 95\%: 11,05-12,97). En el análisis por ciudades, Barranquilla presentó la frecuencia más alta (16,22\%; IC 95\%: 14,58-18,01) y Bogotá, la más baja (10,75; IC 95\%: 9,30-12,38). Para los síntomas evaluados con el cuestionario GERDQ, la prevalencia estimada fue: pirosis, 13,6\% (IC 95\%: 12,50-14,60); regurgitación, 16,9\% (IC 95\%: 15,74-17,99); epigastralgia, 16,67\% (IC 95\%: 15,54-17,80): náuseas, 11,4\% (IC 95\%: 10,46-12,35); dificultad para dormir por presentar pirosis o regurgitación, 8,17\% (IC 95\%: 7,36-8,97); y consumo de medicamentos adicionales a los formulados por el médico, 6,68\% (IC 95\%: 6,01-7,35). El sexo femenino, el vivir en Barranquilla o Medellín y el presentar una comorbilidad se asociaron estadísticamente con la presencia de reflujo.

Conclusión: la prevalencia de síntomas de reflujo en cuatro ciudades capitales de Colombia, medida con el cuestionario GERDQ, de 11,98\% (IC 95\%: 11,05-12,97), es similar a la reportada en otros países de Latinoamérica, siendo las comorbilidades (particularmente hipertensión arterial [HTA]) el factor que más se asoció con esta condición en todos los estratos del estudio.

Palabras clave

Prevalencia, síntomas, reflujo gastroesofágico, GERDQ, factores asociados. 


\begin{abstract}
Problem: Gastroesophageal reflux is a physiological process that can become pathological in some people. It can cause discomfort and esophageal and extra-esophageal injuries and can affect the quality of life of anyone affected by it. Currently there are no statistics on the frequency of this condition in Colombia.

Objective: The objective of this study was to use the GerdQ questionnaire to quantify the prevalence of gastroesophageal reflux disease symptoms in adults in four major cities of Colombia and to explore the association of sociodemographic and anthropometric variables with this disease.

Methodology: This was a cross-sectional population-based study of 6,842 people between the ages of 18 years and 80 in Bogotá, Cali, Medellín and Barranquilla. The sample was selected by stratified random sampling. A telephone survey was conducted that included the GERD Q questionnaire for the identification of reflux symptoms within the week prior to an interview. The prevalence of reflux by socioeconomic strata was calculated using a cut-off point of 8 or more points. Comparisons were made among strata using the $x 2$ test. Associations of sociodemographic and anthropometric variables were explored through bivariate analysis and logistic regression models.

Results: We obtained 6,842 surveys. The overall estimated prevalence of reflux symptoms was $11.98 \%$ $(95 \% \mathrm{Cl}=11.05 \%-12.97 \%)$. The city of Barranquilla presented the highest frequency of $16.22 \%(95 \% \mathrm{Cl}=$ $14.58 \%-18.01 \%)$ while Bogotá had the lowest of $10.75 \%(95 \% \mathrm{Cl}=9.30 \%-12.38 \%)$. For symptoms evaluated with GERD-Q, the estimated prevalences were: heartburn $13.6 \%(95 \% \mathrm{Cl}=12.50 \%-14.60 \%)$, regurgitation $16.9 \%(95 \% \mathrm{Cl}=15,74 \%-17.99 \%)$, epigastralgia $16.67 \%(95 \% \mathrm{Cl}=15.54 \%-17.80 \%)$, nausea $11.4 \%$ $(95 \% \mathrm{Cl}=10.46 \%-12.35 \%)$, difficulty sleeping due to heartburn or regurgitation $8.17 \%(95 \% \mathrm{Cl}=7.36 \%$ $-8.97 \%)$ and consumption of medications additional to those formulated by the physician $6.68 \%(95 \% \mathrm{Cl}=$ $6,01 \%-7.35 \%)$. Women living in Barranquilla or Medellin, had statistically significant levels of comorbidities associated with reflux.

Conclusion: The prevalence of reflux symptoms in four important Colombian cities measured with the GerdQ questionnaire was $11.98 \%(95 \% \mathrm{Cl}=11.05-12.97)$ which is similar to prevalences reported in other Latin American countries. Comorbidities (particularly hypertension) are the factor that was most frequently associated with this condition in all strata of the study.

Keywords

Prevalence, symptoms, gastroesophageal reflux, Gerd Q, associated factors.
\end{abstract}

\section{INTRODUCCIÓN}

La enfermedad por reflujo gastroesofágico (ERGE) afecta un gran número de personas en todo el mundo y constituye una importante carga en los sistemas de salud por el alto número de consultas que genera, los costos de los medicamentos, la reducción de la calidad de vida $(1,2)$ y el impacto en la productividad laboral (3-7). En América del Norte y Europa, en estudios poblacionales que miden la presencia de síntomas semanalmente, se ha estimado una prevalencia de reflujo en adultos que varía entre el $10 \%$ y el 20\% (8). Una cifra similar se observa en América Latina, donde la prevalencia estimada de síntomas como pirosis y regurgitación, experimentados al menos semanalmente, ha sido informada entre el 11,9\% y el 31,3\% (9), mientras que en Asia la prevalencia descrita oscila entre el 2,5\% y el 6,7\% (10). En países occidentales, se ha estimado que el $5 \%$ de la consulta en atención primaria corresponde a la atención de reflujo gastroesofágico (5), mientras que en Brasil, la probabilidad de asistir a consulta en las personas con síntomas de reflujo semanal es dos veces más alta que aquellos con síntomas menos frecuentes (1).
Mundialmente se han realizado diferentes estudios tanto a nivel poblacional como hospitalario para conocer la frecuencia de ERGE, su asociación con factores sociodemográficos, ambientales, genéticos, entre otros (1, 11-28), y su relación como posible factor de riesgo para el desarrollo de enfermedades como el adenocarcinoma de esófago, el carcinoma del cardias y el carcinoma escamocelular de esófago (29). Sin embargo, es difícil la comparabilidad entre los estudios dada la alta heterogeneidad entre ellos, las diferentes definiciones de la enfermedad, la variabilidad en la metodología y la diversidad de los instrumentos usados para el diagnóstico, que van desde la aplicación de cuestionarios estandarizados como el GERDQ (gastroesophageal reflux disease questionnaire) (11) y otros creados por los propios investigadores (algunas veces no validados), hasta la realización de pruebas mucho más específicas como la endoscopia, la pH-metría o la manometría esofágica, que son difíciles de llevar a cabo en estudios poblacionales dada su complejidad operativa y altos costos. El GERDQ permite la identificación de síntomas típicos de reflujo y muestra una sensibilidad del $65 \%$ y una especificidad del $71 \%$, datos que se asemejan al diagnóstico por gastroente- 
rólogo (11). Esta herramienta ha sido validada en nuestra población, observándose una correlación del $89 \%$ con el diagnóstico realizado por gastroenterólogo cuando el puntaje obtenido es mayor de 8 puntos (30).

En Colombia, aunque la ERGE es una causa muy frecuente de consulta tanto a nivel primario como consulta especializada, no disponemos de información que nos permita sugerir una posible prevalencia ni sus probables asociaciones. Conocer la prevalencia poblacional del reflujo gastroesofágico en nuestro país es útil para la propuesta y direccionamiento de actividades que ayuden al control de la enfermedad, así como para promover actividades educativas de diagnóstico y de manejo de esta patología en Colombia. El objetivo de este estudio es cuantificar la prevalencia de síntomas de ERGE en los adultos de cuatro ciudades de Colombia utilizando el cuestionario GERDQ, y explorar las asociaciones con variables sociodemográficas y antropométricas.

\section{METODOLOGÍA}

Se llevó a cabo un estudio observacional tipo corte transversal con base poblacional, en cuatro de las principales ciudades de Colombia: Barranquilla, Bogotá, Cali y Medellín, en el período de febrero a julio de 2014. El diseño planteado fue un muestreo aleatorio estratificado.

\section{Cálculo del tamaño de la muestra}

De acuerdo con las proyecciones poblacionales del Departamento Administrativo Nacional de Estadística (DANE) con base en el censo 2005, se estimaron 38196000 personas para el año 2013, de las cuales, el $72 \%$ residen en zona urbana (27 501120 individuos). A partir de esta población, se calculó un tamaño de muestra de 1537 individuos por cada ciudad, para una prevalencia esperada de síntomas de reflujo del $20 \%$, con precisión absoluta del $2 \%$ alrededor del estimador y nivel de confianza del $95 \%$. Considerando un efecto de no respuesta de estrategia telefónica de 1,3, se ajustó el cálculo a 1998 personas por ciudad, para un total de 7992 individuos. Teniendo en cuenta que para poblaciones de tamaño finito mayores de 500000 habitantes no es necesario incrementar el tamaño de muestra para sostener los parámetros de precisión, exactitud y confianza, y que para cada estrato (ciudad) de esta investigación el tamaño de la población de estudio es mayor que este número (Bogotá: 5382 729; Medellín: 1811 680; Cali: 1621 057; Barranquilla: 821 907), el tamaño de muestra que se manejará para cada estrato tendrá la misma magnitud (1998 personas).

Si se considera que la obesidad es una variable que puede sesgar el estimador global del estrato (la presencia de obesidad se asocia con mayor frecuencia de síntomas de reflujo), este tamaño de muestra permite conservar una precisión del 5\% alrededor del estimador de síntomas de reflujo para el subgrupo de personas no obesas, asumiendo una prevalencia de obesidad del $20 \%$.

El cálculo del tamaño de muestra fue realizado en el software PASS $12^{\circ}$.

La selección de los estratos tuvo en cuenta la necesidad de incorporar ciudades capitales que representaran grupos sociales y culturales diferentes dentro del país.

Se incluyeron hombres y mujeres entre los 18 y 79 años de edad, residentes en alguna de estas cuatro ciudades y que aceptaran participar en el estudio. Se excluyeron las mujeres embarazadas, así como las personas con neoplasia digestiva actual y cirugía en tracto digestivo alto, dado que en estas condiciones se pueden presentar síntomas gastrointestinales que podrían generar sobrestimación de la prevalencia de ERGE; adicionalmente se excluyeron las personas con nacionalidad diferente a la colombiana.

A todas las personas que aceptaron participar se les aplicó una encuesta telefónica que incluyó variables sociodemográficas y antropométricas y el cuestionario GERDQ (30), el cual se encuentra validado para Colombia. Esta herramienta consta de 4 predictores positivos de ERGE: ardor retroesternal (pirosis) y regurgitación, alteración del sueño por estos dos síntomas y uso de medicamentos adicionales para producir alivio de estos síntomas, y dos predictores negativos de ERGE: dolor epigástrico y náuseas (11).

\section{Procedimientos}

A partir de un diseño preliminar del instrumento que se empleó para la encuesta telefónica, se realizó un estudio piloto para ajustar el contenido y secuencia de las preguntas, así como para estandarizar el método de aplicación del instrumento. Este estudio se efectuó con 20 voluntarios, mayores de edad, de cada una de las ciudades que conformaron los estratos del estudio.

Una vez obtenida una versión definitiva del instrumento de medición, se contrataron y entrenaron, por parte de uno de los investigadores, técnicos profesionales en salud, y por medio de un centro de llamadas se hicieron las llamadas telefónicas a las unidades de vivienda seleccionadas en el muestreo. Se aplicaron los criterios de selección propuestos en este estudio a la persona que atendió la llamada telefónica. En caso de que no cumplir dichos criterios, se exploró la posibilidad de que otra persona que resida en la misma unidad participara en el estudio. Se realizó la encuesta que incluyó las variables descritas anteriormente; para las variables antropométricas (peso y talla) se ha demostrado un coeficiente de correlación intraclase entre el autorreporte y la medición directa sobre el sujeto de 0,956 para peso (IC95\%: 0,95-0,97) y de 0,953 para estatura (IC95\%: 0,91-0,97), 
razón por la cual se considera tolerable el grado de error del dato brindado por los participantes en la encuesta telefónica.

Se llevó a cabo un control de calidad en el $10 \%$ del total de las encuestas llamando nuevamente a los participantes y verificando la concordancia de la información.

La información fue recolectada en formato físico electrónico, que permitía la captura de la información, y fue grabada en una base de datos en Access; la grabación se hizo semanalmente, en lotes de 500 formatos, por duplicado, por digitadores independientes. Una vez completada la grabación de todos los formatos, se realizó una confrontación entre los registros provenientes de cada digitador y se ajustaron las inconsistencias mediante la verificación en los formatos respectivos. Una vez obtenida la base limpia definitiva, se procedió a la exportación y alistamiento de las subbases en el programa Stata para proceder a los análisis estadísticos requeridos.

\section{Aspectos éticos}

En el presente estudio se respetaron y cumplieron los principios para la investigación en humanos enunciados en la declaración de Helsinki de la Asociación Médica Mundial, y las pautas éticas contempladas por el Consejo de Organizaciones Internacionales de las Ciencias Médicas (CIOMS) y el Informe de Belmont. Así mismo se aceptaron los lineamientos jurídicos y éticos descritos en la Resolución 8430 de 1993 del Ministerio de Salud de Colombia y demás normatividad nacional.

Por tratarse de un estudio observacional en el que solo se aplicaron encuestas telefónicas, y no se realizó ninguna intervención o modificación intencionada de las variables biológicas, fisiológicas, psicológicas o sociales de los individuos que participaron en el estudio, la Resolución 8430 de 1993 del Ministerio de Salud de Colombia lo clasifica como investigación sin riesgo, razón por la cual no se consideró necesario el consentimiento informado por escrito. No obstante, el protocolo fue sometido para evaluación ante el Comité de Ética en Investigación del Hospital Santa Clara, que aprobó el protocolo según un acta de marzo de 2014. El Comité recomendó la toma de consentimiento al inicio de la encuesta, lo que se llevó a cabo. El acta reposa en las oficinas de la Asociación Colombiana de Gastroenterología.

La confidencialidad de los participantes se garantizó restringiendo el acceso a los datos de identificación únicamente a las personas encargadas de hacer las encuestas y al coordinador del estudio. En los instrumentos de recolección de datos se registró el código de identificación asignado y en ningún caso se revelará la identidad de los individuos incluidos en el estudio.
Los resultados de la investigación serán publicados en revistas de carácter académico y científico y corresponden a datos generales y no a un participante en particular.

A las personas que decidieron participar en este estudio y a las cuales se les identifique que presentaron reflujo se les brindó la orientación para que asistieran a consulta médica y de esta manera recibieran el tratamiento necesario para su enfermedad.

\section{Análisis estadístico}

Se efectuó el análisis descriptivo de la población estimando frecuencias absolutas y relativas para las variables cualitativas, y medidas de tendencia central y de dispersión para las variables cuantitativas. Se estimó la prevalencia de ERGE junto con su correspondiente intervalo de confianza del 95\% a partir del cuestionario GERDQ, donde se establece como punto de corte 8 puntos o más para el diagnóstico de reflujo; así mismo, a partir de esta herramienta, se obtuvo la prevalencia en la última semana de los síntomas de esta enfermedad.

Para los análisis de asociación entre reflujo y variables sociodemográficas y antropométricas se efectuaron inicialmente análisis bivariados utilizando pruebas ANOVA para variables continuas o $x^{2}$ para variables categóricas. Para todas las pruebas de hipótesis se consideraron valores de significación del $5 \%$ y pruebas a dos colas. Ulteriormente se desarrollaron modelos de asociación para evaluar y cuantificar la relación entre variables antropométricas y sociodemográficas y la presencia de reflujo; para esto se plantearon modelos de regresión logística binaria y ordinal en donde se tomaron como estimadores los odds ratio (OR) correspondientes a los coeficientes de regresión transformados.

Los análisis se realizaron en el software Stata $13^{\circ}$, donde se incorporó para su cálculo el efecto del diseño muestral.

\section{RESULTADOS}

Se realizaron 9222 encuestas de las cuales se incluyeron 6842 en el análisis. Las razones de exclusión fueron: 1645 individuos decidieron no participar; 195 no cumplieron con el criterio de edad; 113 tenían cirugía en esófago o estómago; 9 tenían diagnóstico de cáncer; 35 no eran colombianos; y 36 mujeres estaban en embarazo.

Respecto a las características de los sujetos incluidos en el estudio (tabla 1): el promedio de edad fue de 44,07 años (IC 95\%: 43,58-44,56); la mayoría eran mujeres; el nivel de escolaridad más frecuentemente encontrado fue el de secundaria completa; el estado civil más reportado fue casado; y la mayoría de la población vivía en estratos 2 y 3. 
En cuanto al estado nutricional, se observó que la mayoría de la población tenía peso normal, aunque un importante porcentaje presentó sobrepeso 30,5 (IC 95\%: 29,1-31,8). El 9,5\% (IC 95\%: 8,6-10,4) de los encuestados consumía cigarrillo. El 15,8\% (IC 95\%: 14,7-16,9) refirió haber consumido licor en la última semana antes de la encuesta y el 27,1\% (IC 95\%: 25,8-28,5) presentó una comorbilidad, siendo la hipertensión, la diabetes y las enfermedades en el aparato digestivo las más frecuentes en hombres, mientras que en mujeres, las tres comorbilidades más informadas, en orden descendente, fueron la hipertensión, las patologías tiroideas (hipertiroidismo o hipotiroidismo) y las enfermedades en aparato digestivo (tabla 2).

La prevalencia general de síntomas de reflujo para la población, de acuerdo con el cuestionario GERDQ, fue la siguiente: pirosis, 13,6\% (IC 95\%: 12,50-14,60); regurgitación, 16,9\% (IC 95\%: 15,74-17,99); epigastralgia, 16,67 (IC 95\%: 15,54-17,80); náuseas, 11,4\% (IC 95\%: 10,46-12,35); dificultad para dormir por presentar pirosis o regurgitación, $8,17 \%$ (IC 95\%: 7,36-8,97); y consumo de medicamentos adicionales a los formulados por el médico, 6,68\% (IC 95\%: 6,01-7,35). La prevalencia de síntomas de reflujo por ciudades se presenta en la figura 1 , donde se observa que a excepción de la pirosis, que se presentó con mayor frecuencia en Medellín (14,6\%; IC 95\%: 12,93-16,29), los otros síntomas fueron más frecuentes en Barranquilla.

La prevalencia de síntomas de reflujo para toda la población fue de 11,98\% (IC 95\%: 11,05-12,97), siendo Barranquilla la ciudad con la cifra más alta (16,22\%; IC

Tabla 1. Características sociodemográficas de los sujetos incluidos en el estudio

\begin{tabular}{|c|c|c|c|c|}
\hline Variable & Categorías & Proporción & Error estándar & Intervalo de confianza $95 \%$ \\
\hline Sexo & Mujer & 72,4 & 0,700 & $71,1-73,8$ \\
\hline \multirow[t]{4}{*}{ Edad } & $18-30$ & 25,9 & 0,676 & $24,6-27,2$ \\
\hline & $31-45$ & 27,8 & 0,692 & $26,5-29,2$ \\
\hline & $46-55$ & 20,0 & 0,621 & $18,8-21,2$ \\
\hline & $>55$ & 26,3 & 0,678 & $24,9-27,6$ \\
\hline \multirow[t]{6}{*}{ Estado civil } & Casado & 38,2 & 0,748 & $36,7-39,6$ \\
\hline & Soltero & 34,9 & 0,740 & $33,5-36,4$ \\
\hline & Unión libre & 15,6 & 0,564 & $14,5-16,7$ \\
\hline & Divorciado / separado & 5,1 & 0,342 & $4,5-5,8$ \\
\hline & Viudo & 4,7 & 0,311 & $4,1-5,3$ \\
\hline & Sin dato & 1,5 & 0,172 & $1,1-1,8$ \\
\hline \multirow[t]{9}{*}{ Escolaridad } & No ha estudiado & 1,3 & 0,173 & $0,99-1,7$ \\
\hline & Primaria incompleta & 3,8 & 0,264 & $3,3-4,3$ \\
\hline & Primaria completa & 12,9 & 0,514 & $11,9-13,9$ \\
\hline & Secundaria incompleta & 8,8 & 0,419 & $8,0-9,7$ \\
\hline & Secundaria completa & 29,4 & 0,697 & $28,1-30,8$ \\
\hline & Técnico / tecnólogo & 17,5 & 0,597 & $16,3-18,6$ \\
\hline & Universitaria incompleta & 5,8 & 0,362 & $5,1-6,5$ \\
\hline & Universitaria completa & 17,2 & 0,605 & $16,0-18,4$ \\
\hline & Posgrado & 1,7 & 0,215 & $1,3-2,1$ \\
\hline Estrato & 1 y 2 & 48,7 & 0,775 & $47,2-50,2$ \\
\hline \multirow[t]{2}{*}{5 y 6} & 3 y 4 & 46,0 & 0,778 & $44,5-47,5$ \\
\hline & 5,3 & 0,340 & $4,6-5,9$ & \\
\hline \multirow[t]{4}{*}{ Estado nutricional } & Bajo peso & 3,1 & 0,265 & $2,6-3,7$ \\
\hline & Peso normal & 54,7 & 0,766 & $53,2-56,2$ \\
\hline & Sobrepeso & 30,5 & 0,709 & $29,1-31,8$ \\
\hline & Obesidad & 11,7 & 0,477 & $10,7-12,6$ \\
\hline
\end{tabular}


Tabla 2. Proporción de comorbilidades informadas en hombres y mujeres

\begin{tabular}{lccc}
\hline \multicolumn{1}{c}{ Comorbilidad } & Hombres\% (IC 95\%) & Mujeres\% (IC 95\%) & Total\% (IC 95\%) \\
\hline Hipertensión & $33,82(28,18-39,96)$ & $34,36(31,43-37,43)$ & $34,25(31,63-36,97)$ \\
Enfermedades del tiroides & $5,49(2,98-9,89)$ & $17,29(14,88-19,98)$ & $14,84(12,82-17,13)$ \\
Enfermedades del aparato digestivo & $11,49(7,9-16,41)$ & $13,36(11,38-15,63)$ & $12,97(11,19-14,99)$ \\
Diabetes & $13,1(9,38-18,01)$ & $8,72(7,08-10,69)$ & $9,63(8,07-11,44)$ \\
Enfermedades del sistema osteomuscular y tejido conectivo & $7,56(4,66-12,03)$ & $9,56(7,82-11,65)$ & $9,15(7,6-10,98)$ \\
Dislipidemia & $6,11(3,59-10,22)$ & $6,67(5,26-8,44)$ & $6,56(5,28-8,13)$ \\
Enfermedades del sistema respiratorio & $7,34(4,47-11,84)$ & $5,62(4,27-7,37)$ & $5,98(4,7-7,58)$ \\
Otra enfermedad cardiovascular & $4,82(2,62-8,69)$ & $3,58(2,55-5,01)$ & $3,84(2,85-5,15)$ \\
Enfermedades mentales & $1,58(0,61-4,02)$ & $2,66(1,85-3,79)$ & $2,43(1,74-3,39)$ \\
\hline
\end{tabular}

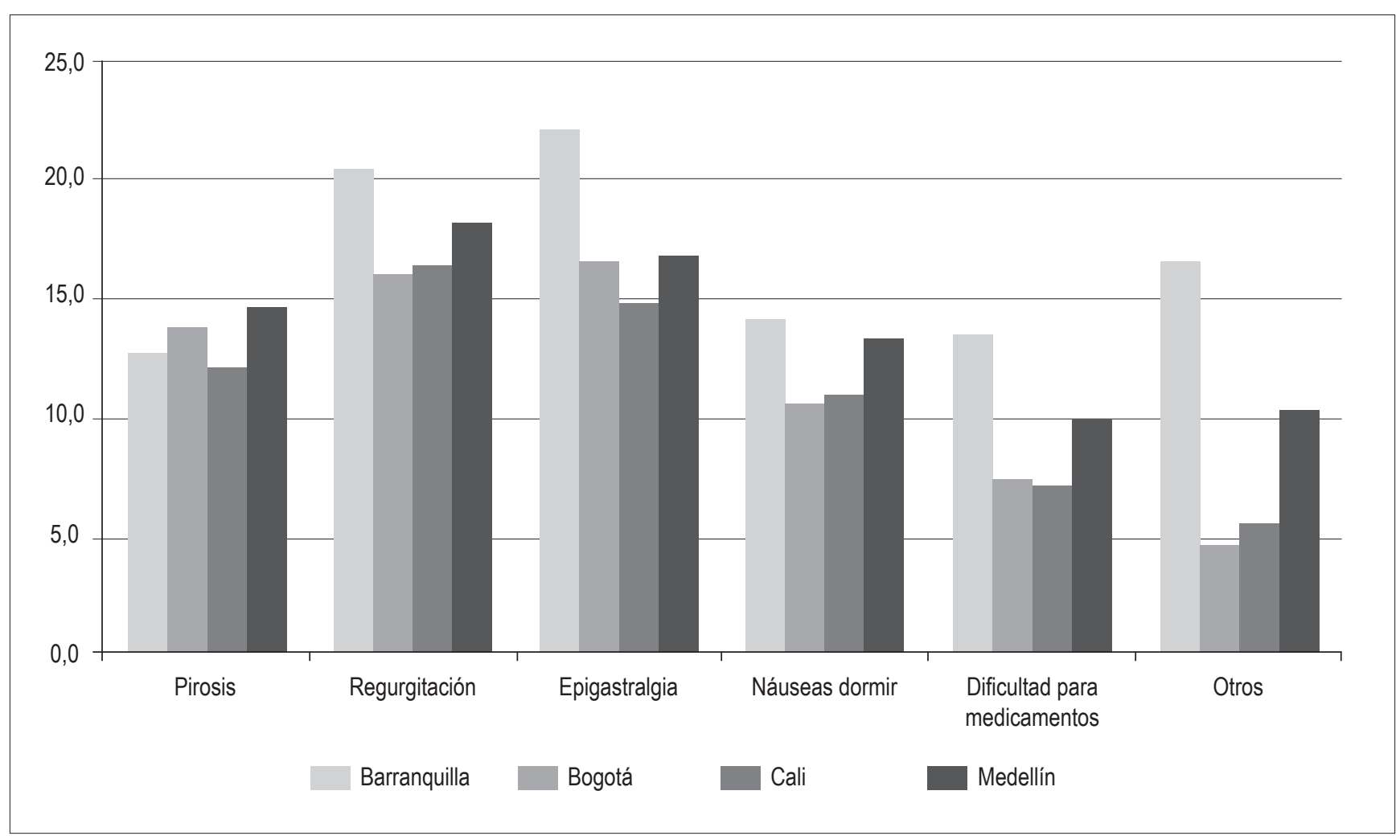

Figura 1. Prevalencia de síntomas por ciudad.

95\%: 14,58-18,01), seguida de Medellín (14,06\%; IC 95\%: 12,49-15,79), Cali (11,6\%; IC 95\%: 10,17-13,19) y Bogotá (10,75\%; IC 95\%: 9,30-12,38).

En el análisis multivariado se observó que los factores estadísticamente asociados con la presencia de reflujo para la población en general fueron: ser mujer, vivir en Barranquilla o Medellín (comparado con Bogotá) y tener una comorbilidad. Cuando se hizo este mismo análisis estratificado por ciudades, se observó que además de la comorbilidad, que es un factor de riesgo para tener reflujo en todas las ciudades, en
Barranquilla y Medellín las personas con obesidad presentaron un riesgo mayor de tener enfermedad por reflujo comparadas con aquellas con peso normal (OR: 1,89; IC 95\%: 1,28-2,77; y OR: 1,75; IC 95\%: 1,15-2,67; respectivamente). En Cali, las mujeres presentaron más riesgo que los hombres (OR: 1,91; IC 95\%: 1,26-2,89), así como los fumadores comparados con los no fumadores (OR: 1,87; IC 95\%: 1,07$3,28)$. Además se encontró que vivir en estratos 3 y 4 es un factor protector comparado con vivir en estratos 5 y 6 (OR: 0,48; IC 95\%: 0,26-0,87) (tabla 3). 
Tabla 3. Factores asociados con la presencia de reflujo. Análisis ajustado general y estratificado por ciudades

\begin{tabular}{|c|c|c|c|}
\hline Variable & $\begin{array}{c}\text { Odds } \\
\text { ratio } \\
\text { ajustado }\end{array}$ & $\begin{array}{l}\text { Valor } \\
\text { de } p\end{array}$ & $\begin{array}{c}\text { Intervalo de } \\
\text { confianza } \\
\text { del } 95 \%\end{array}$ \\
\hline Sexo femenino & 1,37 & 0,008 & $1,08-1,73$ \\
\hline Barranquilla & 1,55 & $<0,001$ & $1,25-1,92$ \\
\hline Medellín & 1,30 & 0,019 & $1,04-1,62$ \\
\hline Padece de otra enfermedad & 2,06 & $<0,001$ & $1,67-2,54$ \\
\hline \multicolumn{4}{|l|}{ Análisis estratificado por ciudades } \\
\hline \multicolumn{4}{|l|}{ Barranquilla } \\
\hline Obesidad & 1,89 & 0,001 & $1,28-2,77$ \\
\hline Padece de otra enfermedad & 2,13 & $<0,001$ & $1,60-2,84$ \\
\hline \multicolumn{4}{|l|}{ Bogotá } \\
\hline Padece de otra enfermedad & 2,07 & $<0,001$ & $1,41-3,05$ \\
\hline \multicolumn{4}{|l|}{ Cali } \\
\hline Sexo femenino & 1,91 & 0,002 & $1,26-2,89$ \\
\hline Estrato 3 y 4 & 0,48 & 0,016 & $0,26-0,87$ \\
\hline Consumo de cigarrillo & 1,87 & 0,029 & $1,07-3,28$ \\
\hline Padece de otra enfermedad & 2,24 & $<0,001$ & $1,56-3,20$ \\
\hline \multicolumn{4}{|l|}{ Medellín } \\
\hline Obesidad & 1,75 & 0,009 & $1,15-2,67$ \\
\hline Padece de otra enfermedad & 1,93 & $<0,001$ & $1,44-2,64$ \\
\hline
\end{tabular}

\section{DISCUSIÓN}

La ERGE resulta del flujo retrógrado del contenido gástrico en el esófago $u$ órganos adyacentes a este, que produce un espectro variable de signos y síntomas esofágicos y/o extraesofágicos, con o sin daño tisular. El consenso de Montreal de 2006 definió la ERGE como una condición que se desarrolla cuando el reflujo del contenido gástrico causa síntomas molestos o complicaciones (31).

La ERGE como entidad clínica tiene un espectro variado de presentaciones y los síntomas pueden no correlacionarse con los hallazgos endoscópicos (31-33) y los pacientes pueden presentar combinación de manifestaciones:

- Síntomas típicos de reflujo con hallazgos endoscópicos de lesión mucosa esofágica

- Síntomas típicos de reflujo con endoscopia normal

- Sin síntomas típicos de reflujo, pero con evidencia endoscópica de lesión en la mucosa

- Síntomas atípicos tales como dispepsia, tos, asma, entre otros $(31,32)$.

Los síntomas que se han considerado típicos de la ERGE son la pirosis y la regurgitación y originalmente se estimó para estos síntomas una sensibilidad del $78 \%$ y una especificidad del $60 \%$ para la presencia de $\operatorname{ERGE}(31,34)$.
Los síntomas relacionados con la ERGE se catalogan como molestos cuando afectan adversamente la calidad de vida del paciente en relación con su salud. Los síntomas que no son considerados molestos por el paciente no deben diagnosticarse como ERGE. En los estudios poblacionales se consideraron como molestos los síntomas leves experimentados 2 o más días por semana, o los síntomas moderados o incluso severos experimentados al menos 1 día por semana; sin embargo, los umbrales de frecuencia sintomática utilizados para definir la ERGE varían ampliamente entre los distintos estudios epidemiológicos dirigidos a evaluar la prevalencia de esta enfermedad $(9,31)$.

El presente estudio utilizó el cuestionario GERDQ, un instrumento diagnóstico basado en síntomas, diseñado para evaluar la prevalencia de la enfermedad. Este instrumento fue validado en nuestro medio $(11,30)$ y utiliza como punto de corte un puntaje de 8 , lo cual significa que quienes tengan un puntaje igual o mayor de 8 tienen alta probabilidad de padecer ERGE y que aquellos con un puntaje menor tienen baja probabilidad. Un punto de corte de 8 tiene la mayor especificidad $(71,4 \%)$ y sensibilidad (64,6\%), y en diversos estudios se ha demostrado que con el cuestionario GERDQ se alcanza una exactitud diagnóstica similar a la del gastroenterólogo (11,35-37).

La prevalencia de reflujo en cuatro ciudades capitales de Colombia, diagnosticada con el cuestionario GERDQ es similar a la reportada en varios países de América Latina, siendo una de las más bajas en esta región $(9,12,38)$. La discordancia con países como Venezuela, Brasil y México, donde la frecuencia de ERGE reportada es mayor, puede explicarse por las diferencias metodológicas con estos estudios, dado que la prueba utilizada para el diagnóstico de la enfermedad, el tipo de población, la metodología de recolección de datos y el tiempo de evaluación de síntomas no siempre son los mismos que se plantearon en nuestro estudio $(1,19,22,39,40)$. Sin embargo, llama la atención que en un estudio realizado por Moraes-Filho y colaboradores (41), en población urbana brasileña con una muestra poblacional importante $(n=13959)$, con encuesta persona a persona, se encontró una prevalencia semanal de pirosis cercana a la nuestra.

Los resultados del análisis de la relación entre variables sociodemográficas y antropométricas con la frecuencia de reflujo en las cuatro ciudades incluidas en el estudio mostraron un patrón propio de nuestra población, que coincide con algunos de los factores de riesgo hallados en otros países de la región. Un ejemplo de esto es la asociación del reflujo con el sexo femenino, la cual ha sido documentada en estudios de América Latina $(19,21,22,24,39-42)$, pero difiere de los resultados de estudios realizados en poblaciones de Estados Unidos, Europa e Irán, donde no se ha encontrado esta aso- 
ciación (16-18, 26-28), y de estudios en China, donde la frecuencia de reflujo es más alta en hombres que en mujeres $(14,25)$. Cuando se evalúan los síntomas de ERGE, la prevalencia de géneros es muy variable en los diferentes países como es resaltado aquí; sin embargo, cuando se evalúan las complicaciones de la ERGE (variables más específicas), los datos consistentemente indican que la enfermedad es más frecuente en hombres. Una posibilidad que explicaría la mayor prevalencia en mujeres en este estudio podría ser que en ellas, los síntomas funcionales esofágicos son más frecuentes que en los hombres. Diferentes estudios han demostrado que entidades como la pirosis funcional, el globo faríngeo y el dolor torácico no cardiogénico son más prevalentes en mujeres (43-47).

Con respecto a la asociación entre presentar una comorbilidad y tener reflujo, predominaron el informe de las enfermedades cardiovasculares y del tiroides, en contraste con otros estudios donde se ha observado que las comorbilidades gastrointestinales como el síndrome de intestino irritable y la úlcera péptica están asociadas con mayor frecuencia de síntomas de reflujo (43), así como la disfagia y la dispepsia (17). Igualmente se ha demostrado asociación estadística con otras enfermedades no digestivas como tos, enfermedad de la vesícula biliar, sinusitis, dolor en el tórax (43), e incluso enfermedades mentales como depresión y ansiedad (15).

Por el contrario, la asociación entre el estado nutricional, el consumo de cigarrillo o el consumo de alcohol y la enfermedad por reflujo informada previamente por diferentes autores $(1,16-18,21,24-26,42-45)$ no fue significativa en nuestro estudio, lo cual podría explicarse por la variabilidad en la medición de estas variables.

Los datos generados en la presente investigación brindan una estimación confiable de la prevalencia de reflujo en las cuatro ciudades donde se aplicaron las encuestas. Esto por el gran tamaño de la muestra y el uso de una herramienta que tiene una alta correlación con el diagnóstico realizado por un gastroenterólogo y que ha sido validada en varios países, así como también población colombiana $(11,20$, 30 ), y que además permite la diferenciación de los síntomas específicos de reflujo y del grado de severidad de esta enfermedad (11). Otra fortaleza de este estudio es la inclusión de ciudades representativas de diferentes agrupaciones socioculturales del país, lo que permite tener una aproximación de la prevalencia de reflujo en Colombia. No obstante, existen debilidades metodológicas, como las llamadas a teléfonos fijos, que excluyen a una parte de la población, especialmente en zona rural. También debemos considerar un evidente sesgo de selección en cuanto al predominio del género femenino en la muestra y la importante prevalencia de comorbilidad cardiovascular cercana al
$40 \%$, que posiblemente se acompañe de polimedicación, lo cual podría modificar la prevalencia de síntomas de ERGE.

Desde el punto de vista de salud pública, este aporte al conocimiento de la epidemiologia de la ERGE en nuestro país nos permite hacer la cuantificación y planificación de recursos destinados a la atención de este tipo de patologías y también la valoración de las variables explicativas o los factores de riesgo que puedan ser intervenidos de manera preventiva. De otra parte, en la práctica clínica, la utilización de cuestionarios como el GERDQ, validados y con una probabilidad pretest satisfactoria, permiten su recomendación en aras de la racionalidad en el uso de los recursos diagnósticos, particularmente en la atención primaria $(30,46)$. El desarrollo de estudios adicionales de prevalencia utilizando instrumentos validados permitirá el seguimiento y la vigilancia del reflujo gastroesofágico, en particular buscando modificar los factores de riesgo.

\section{Conflictos de interés}

El presente estudio fue realizado por el Comité de Investigaciones de la Asociación Colombiana de Gastroenterología, con fondos provenientes de las siguientes compañías farmacéuticas: Bussié, Galeno, Grupo Farma, Reckitt Benckiser, Takeda y Tecnoquímicas-MK; el formulario GERDQ fue facilitado por Laboratorios AstraZeneca.

\section{REFERENCIAS}

1. Pereira-Lima J, Blaya C, Rynkowski CB, et al. Populationbased study on the prevalence of gastroesophageal reflux disease in Porto Alegre, Rio Grande do Sul. GED Gastroenterol Endosc Dig. 2014;19(2):73-8.

2. Wiklund I. Review of the quality of life and burden of illness in gastroesophageal reflux disease. Dig Dis. 2004;22(2):108-14.

3. Enck P, Dubois D, Marquis P. Quality of life in patients with upper gastrointestinal symptoms: results from the Domestic/ International Gastroenterology Surveillance Study (DIGEST). Scand J Gastroenterol. 1999;34(231):48-54.

4. Haycox A, Einarson T, Eggleston A. The health economic impact of upper gastrointestinal symptoms in the general population: results from the Domestic/International Gastroenterology Surveillance Study (DIGEST). Scand J Gastroenterol. 1999;34(231):38-47.

5. Liker H, Hungin P, Wiklund I. Managing gastroesophageal reflux disease in primary care: the patient perspective. J Am Board Fam Pract. 2005; 18(5):393-400.

6. Wahlquist P, Reilly MC, Barkun A. Systematic review: the impact of gastro-oesophageal reflux disease on work productivity. Aliment Pharmacol Ther. 2006;24(2):259-72.

7. Mason J, Hungin AP. Review article: gastro-oesophageal reflux disease--the health economic implications. Aliment Pharmacol Ther. 2005;22 Suppl 1:20-31. 
8. Dent J, El-Serag HB, Wallander M, et al. Epidemiology of gastro-oesophageal reflux disease: a systematic review. Gut. 2005;54(5):710-7.

9. Salis G. Revisión sistemática: epidemiología de la enfermedad por reflujo gastroesofágico en Latinoamérica. Acta Gastroenterol Latinoam. 2011;41(1):60-9.

10. Wong BCY, Kinoshita Y. Systematic review on epidemiology of gastroesophageal reflux disease in Asia. Clin Gastroenterol Hepatol. 2006;4(4):398-407.

11. Jones R, Junghard O, Dent J, et al. Development of the GerdQ a tool for the diagnosis and management of gastro-oesophageal reflux disease in primary care. Aliment Pharmacol Ther. 2009;30(10):1030-8.

12. Dacoll C, Umpierre V, Villa-Gómez M, et al. Prevalencia de la enfermedad por reflujo gastroesofágico en. Uruguay Gastroenterol Hepatol. 2012;35(7):460-7.

13. Eggleston A, Farup C, Meier R. The Domestic/International Gastroenterology Surveillance Study (DIGEST): design, subjects and methods. Scand J Gastroenterol Suppl. 1999;34(231):9-14.

14. Guozong P, Guoming X, Meiyun K, et al. Epidemiological study of symptomatic gastroesophageal reflux disease in China: Beijing and Shanghai. Chin J Dig Dis. 2000;1(1):2-8.

15. Hu WHC, Wong W-M, Lam CLK, et al. Anxiety but not depression determines health care-seeking behaviour in Chinese patients with dyspepsia and irritable bowel syndrome: a population-based study. Aliment Pharmacol Ther. 2002;16(12):2081-8.

16. Isolauri J, Laippala P. Prevalence of symptoms suggestive of gastro-oesophageal reflux disease in an adult population. Ann Med. 1995;27(1):67-70.

17. Locke GR, Talley NJ, Fett SL, et al. Risk factors associated with symptoms of gastroesophageal reflux. Am J Med. 1999;106(6):642-9.

18. Mohammed I, Cherkas LF, Riley SA, et al. Genetic influences in gastro-oesophageal reflux disease: a twin study. Gut. 2003;52(8):1085-9.

19. Moraes-Filho JPP, Chinzon D, Eisig JN, et al. Prevalence of heartburn and gastroesophageal reflux disease in the urban Brazilian population. Arq Gastroenterol. 2014;42(2):122-7.

20. Mungan Z. Prevalence and demographic determinants of gastroesophageal reflux disease (GERD) in the Turkish general population: a population-based cross-sectional study. Turk J Gastroenterol. 2012;23(4):323-32.

21. Nader F, da Costa JSD, Nader GA, et al. Prevalence of heartburn in Pelotas, RS, Brasil: population-based study. Arq Gastroenterol. 2003;40(1):31-4.

22. Peralta-Pedrero ML, Lagunes-Espinosa AL, Cruz-Avelar A, et al. Frequency of gastroesophageal reflux disease in elderly patients attending a family medicine clinic. Rev Med Inst Mex Seguro Soc. 2007;45(5):447-52.

23. Ponce J, Vegazo O, Beltrán B, et al. Prevalence of gastrooesophageal reflux disease in Spain and associated factors. Aliment Pharmacol Ther. 2006;23(1):175-84.

24. Veitía G, Sandoval C, Paredes R, et al. Prevalencia de enfermedad por reflujo gastroesofágico en población adulta del área metropolitana de Caracas, Venezuela. Rev Gastroenterol Nac GEN. 2008;62(2):109-13.

25. Wong WM, Lai KC, Lam KF, et al. Prevalence, clinical spectrum and health care utilization of gastro-oesophageal reflux disease in a Chinese population: a population-based study. Aliment Pharmacol Ther. 2003;18(6):595-604.

26. Kotzan J, Wade W, Yu HH. Assessing NSAID prescription use as a predisposing factor for gastroesophageal reflux disease in a Medicaid population. Pharm Res. 2001;18(9):1367-72.

27. Locke GR, Talley NJ, Fett SL, et al. Prevalence and clinical spectrum of gastroesophageal reflux: a population-based study in Olmsted County, Minnesota. Gastroenterology. 1997; $112(5): 1448-56$.

28. Nouraie M, Razjouyan H, Assady M, et al. Epidemiology of gastroesophageal reflux symptoms in Teheran, Iran: a population- based telephone survey. Arch Iran Med. 2007;10(3):289-94.

29. Lagergren J, Bergstrom R, Lindgren A, et al. Symptomatic gastroesophageal reflux as a risk factor for esophageal adenocarcinoma. N Engl J Med. 1999;340(11):825-31.

30. Santa María M, Jaramillo M, Otero Regino W, et al. Validación del cuestionario de reflujo gastroesofágico “GERDQ” en una población colombiana. Rev Col Gastroenterol. 2013;28(3):199-206.

31. Vakil N, van Zanten SV, Kahrilas P, et al. The Montreal definition and classification of gastroesophageal reflux disease: a global evidence-based consensus. Z Gastroenterol. 2006;45(11):1125-40.

32. Bredenoord AJ. Mechanisms of reflux perception in gastroesophageal reflux disease: a review. Am J Gastroenterol. 2012;107(1):8-15.

33. Vakil N, Malfertheiner, Salis G, et al. An international primary care survey of GERD terminology and guidelines. Dig Dis. 2008;26(3): 231-6.

34. Klauser AG, Schindlbeck NE, Müller-Lissner SA. Symptoms in gastrooesophageal reflux disease. Lancet. 1990;335(8683):205-8.

35. Tytgat GN, McColl K, Tack J, et al. New algorithm for the treatment of gastro-oesophageal reflux disease. Aliment Pharmacol Ther. 2008;27(3):249-56.

36. Jones R, Coyne K, Wiklund I. The gastro-oesophageal reflux disease impact scale: a patient management tool for primary care. Aliment Pharmacol Ther. 2007;25(12):1451-9.

37. Hambleton R. Adaptación de tests para uso en diferentes idiomas y culturas: fuentes de error, posibles soluciones y directrices prácticas. En: Muñiz J. Psicometría. Madrid: Editorial Universitas; 1996. p. 207-238.

38. Chiocca JC, Olmos JA, Salis GB, et al. Prevalence, clinical spectrum and atypical symptoms of gastro-oesophageal reflux in Argentina: a nationwide population-based study. Aliment Pharmacol Ther. 2005;22(4):331-42.

39. De Oliveira SS, dos Santos Ida S, da Silva JFP, et al. Gastroesophageal reflux disease: prevalence and associated factors. Arq Gastroenterol. 2005;42(2):116-21. 
40. Schmulson M, Ortíz O, Santiago-Lomeli M, et al. Frequency of functional bowel disorders among healthy volunteers in Mexico City. Dig Dis. 2006;24(3-4):342-7.

41. Moraes-Filho JP, Chinzon D, Eisig JN, et al. Prevalence of heartburn and gastroesophageal reflux disease in the urban Brazilian population. Arq Gastroenterol. 2005;42(2):122-7.

42. Manterola C, Bustos L, Vial M. Prevalencia de enfermedad por reflujo gastroesofágico en población general urbana adulta. Rev Chil Cir. 2005;57(6):476-82.

43. Ruigómez A, García Rodríguez LA, Wallander M-A, et al. Natural history of gastro-oesophageal reflux disease diagnosed in general practice. Aliment Pharmacol Ther. 2004;20(7):751-60.
44. Watanabe Y, Fujiwara Y, Shiba M, et al. Cigarette smoking and alcohol consumption associated with gastro-oesophageal reflux disease in Japanese men. Scand J Gastroenterol. 2003;38(8):807-11.

45. El-Serag HB, Petersen NJ, Carter J, et al. Gastroesophageal reflux among different racial groups in the United States. Gastroenterology. 2004;126(7):1692-9.

46. Salis G. Revisión sistemática: epidemiología de la enfermedad por reflujo gastroesofágico en Latinoamérica. Acta Gastroenterol Latinoam. 2011;41(1):7-8.

47. Oh JH, Choi MG, Park JM, et al. The clinical characteristics of gastroesophageal reflux disease in patients with laryngeal symptoms who are referred to gastroenterology. Dis Esophagus. 2013;26(5):465-9. 